\title{
Inflationary Quantum Cosmology: General Framework and Exact Bianchi I Solution
}

\author{
Justin Malecki* \\ Perimeter Institute for Theoretical Physics \\ Waterloo, Canada
}

\begin{abstract}
Using the methods of loop quantum gravity, we derive a framework for describing an inflationary, homogeneous universe in a purely quantum theory. The classical model is formulated in terms of the Ashtekar-Sen connection variables for a general subclass of Bianchi class A spacetimes. This formulation then provides a means to develop a corresponding quantum theory. An exact solution is derived for the classical Bianchi type I model and the corresponding semiclassical quantum state is found to also be an exact solution to the quantum theory. The development of a normalizable quantum state from this exact solution is presented and the implications for the normalizability of the Kodama state discussed. We briefly consider some consequences of such a quantum cosmological framework and show that the quantum scale factor of the universe has a continuous spectrum in this model. This result may suggest further modifications that are required to build a more accurate theory. We close this study by suggesting a variety of directions in which to take the results presented.
\end{abstract}

\section{Introduction}

The theory of cosmological inflation [1] has seen continued success since its introduction, most notably in its accurate description of the observed fluctuations of the cosmic microwave background (CMB). Inflation is based on

*email: jjmaleck@physics.ubc.ca 
a classical theory of cosmology which is generally thought to be invalid in regimes close to the big bang where quantum gravitational dynamics must be considered. In this paper, we develop an intrinsically quantum description of inflation to try and understand the role of quantum gravity in the early universe. Our model consists of a spatially homogeneous universe with scalar field matter as this provides the necessary exponential expansion of inflation (with an appropriate choice of scalar field potential).

A theory of quantum cosmology should be based on a more general theory of quantum gravity. Such a theory of quantum gravity that has proved very successful in recent years is the theory of loop quantum gravity. ${ }^{1}$ The starting point for the general theory of loop quantum gravity is the Ashtekar-Sen formulation of general relativity [6, 7] which greatly simplifies the dynamical equations and allows for a well defined quantization of the classical theory. While this quantization procedure is a very general and powerful construction, we follow a simpler route by imposing homogeneity of the underlying spacetime. This allows us to circumvent some of the regularization procedures that are necessary in the full theory.

The application of loop quantum gravity to cosmological scenarios has already seen many intriguing results in the work of Martin Bojowald and others (see [8] for a review of the formulation and the main results). They show that the discreteness of the spectrum of the volume operator leads to many novel effects including a well-defined evolution through the classical big bang singularity 9]. In this paper, we use a similar approach to that of Bojowald to build our inflationary model with the advantage that we are able to find an exact quantum state to describe an inflationary universe.

The framework for this inflationary quantum cosmological model was first introduced in [10. In that study, a quantum state for a flat, isotropic spacetime was derived and discussed. It was found to be normalizable and related to a well-known exact quantum state of the general theory of loop quantum gravity, the Kodama state [11]. The purpose of the following paper is to present a generalization of the previous isotropic model to a broader class of homogeneous spacetimes and to derive exact quantum states for a subclass of these models.

The starting point for building our model is the classical Hamiltonian

\footnotetext{
${ }^{1}$ Thorough, technical introductions to loop quantum gravity are available 2 , 3 as well as shorter, less technical reviews [4, 5], the latter being closely related to the work discussed in this paper.
} 
framework of [10] which is summarized here in section 2, In section 3, we specialize this framework to consider only homogeneous universes and discuss the procedure of quantization. We then focus on a single class of homogeneous universe, Bianchi type I, in section 4 where a family of exact classical and quantum solutions are derived. It is shown in section 4.1 that these solutions can form a normalizable state and we discuss the possible implications for the Kodama state. The specialization of these Bianchi I states to flat, isotropic spacetimes is presented in 5 so as to compare with the analogous state of [10]. We summarize our results in section [6] and suggest some directions for future research. Unless otherwise stated, we work in units in which $c=\hbar=1$ so that the Planck length is $\ell_{p}^{2}=G$.

\section{Classical Hamiltonian Scalar Field Model}

The formalism of loop quantum gravity traditionally begins by describing general relativity as a constrained Hamiltonian theory in terms of AshtekarSen variables [6, 7] before applying the quantization procedure of Dirac [12. Since we are interested in modelling inflation by including a scalar field into our model, it is possible to recast this classical framework into a more familiar Hamiltonian language by using the additional matter degrees of freedom. This derivation was first presented in [10] and so we briefly summarize the procedure here.

The phase space variables used to describe the geometry of a spatial slice of spacetime are a complex, self-dual $S U(2)$ connection $A_{a}^{i}$ and its conjugate momentum $E_{i}^{a}$. Here and throughout we use indices $a, b, c, \ldots \in\{1,2,3\}$ as spatial indices and $i, j, k, \ldots \in\{1,2,3\}$ as $s u(2)$ indices, the latter being raised and lowered by the Kronecker delta $\delta_{i j}$. The momentum $E_{i}^{a}$ is the densitized spatial triad

$$
E_{i}^{a}=\operatorname{det}\left(e_{b}^{j}\right)\left(e^{-1}\right)_{i}^{a}
$$

where $e_{a}^{i}$ is the triad or frame field on the spatial slice and $\left(e^{-1}\right)_{i}^{a}$ its inverse. The connection can be expressed in terms of the three dimensional spin connection $\Gamma_{a}^{i}$ of the frame field and the extrinsic curvature $K_{a}^{b}$ of the spatial slice by

$$
A_{a}^{i}=\Gamma_{a}^{i}+i e_{b}^{i} K_{a}^{b}
$$

The canonical relationship between these phase space variables is given by 
the Poisson bracket

$$
\left\{A_{a}^{i}(x), E_{j}^{b}(y)\right\}=i \ell_{p}^{2} \delta_{a}^{b} \delta_{j}^{i} \delta^{3}(x, y)
$$

where $x$ and $y$ are coordinates on the spatial slice. Since the triad and spin connection are both real quantities, it may be necessary to impose the reality conditions

$$
\begin{aligned}
& A_{a}^{i}+\bar{A}_{a}^{i}=2 \Gamma_{a}^{i} \\
& E_{i}^{a}-\bar{E}_{i}^{a}=0
\end{aligned}
$$

on the phase space variables where denotes complex conjugation.

As in conventional inflationary cosmology, we consider gravity coupled to a scalar field $\phi$ in a potential $V(\phi)$ with conjugate scalar momentum $\pi$. The gauge, diffeomorphism, and Hamiltonian constraints can be written respectively as

$$
\begin{aligned}
G_{i} & =D_{a} E_{i}^{a}=\partial_{a} E_{i}^{a}+\epsilon_{i j}{ }^{k} A_{a}^{j} E_{k}^{a}=0, \\
H_{a} & =E_{i}^{b} F_{a b}^{i}+\pi \partial_{a} \phi=0
\end{aligned}
$$

and

$$
\mathcal{H}=\frac{1}{\ell_{p}^{2}} \epsilon^{i j k} E_{i}^{a} E_{j}^{b}\left(F_{a b k}+\frac{\ell_{p}^{2} V(\phi)}{3} \epsilon_{a b c} E_{k}^{c}\right)+\frac{1}{2} \pi^{2}+\frac{1}{2} E^{a i} E_{i}^{b}\left(\partial_{a} \phi\right)\left(\partial_{b} \phi\right)=0
$$

where $\epsilon_{i j k}$ is the totally antisymmetric, Levi-Civita tensor, $F_{a b k}$ is the curvature of the connection $A_{a}^{i}$, and the cosmological constant $\Lambda$ has been absorbed into the potential $V(\phi)$.

We choose a decomposition of the spacetime manifold $\mathcal{M}$ into $\mathbb{R} \times \mathcal{S}$ by choosing space $\mathcal{S}$ to be defined as a constant $\phi$ hypersurface. The limitations of such a gauge choice are discussed in [10] but are not important for the results of this paper. It is sufficient to know that such a gauge choice is valid during the inflationary phase but may break down after the exponential expansion.

To implement this gauge, we choose a basis of spatial vector fields $\left\{\partial_{a}\right\}_{a=1}^{3}$ such that

$$
\partial_{a} \phi=0
$$

and demand that this constraint be preserved in time by demanding

$$
\left\{\partial_{a} \phi, \mathcal{H}(\tilde{N})\right\}=\partial_{a}(\tilde{N} \pi)=0
$$


where $\mathcal{H}(\tilde{N})=\int_{\mathcal{S}} \tilde{N} \mathcal{H}$ is the integrated Hamiltonian constraint that generates diffeomorphisms in the time direction. Equation (10) implies that the densitized lapse $\tilde{N}$ must take the form

$$
\tilde{N}=\frac{k}{\pi}
$$

where $k$ is a constant, chosen to be $k=\ell_{p}^{-2}$ so that the lapse is dimensionless.

The diffeomorphism and gauge constraints (77) and (6) will be solved automatically when we eventually reduce to the homogeneous models and so we focus only on satisfying the Hamiltonian constraint (8). In light of the time gauge condition (9) we can solve the Hamiltonian constraint for $\pi$ to get

$$
\pi= \pm \sqrt{-\frac{2}{\ell_{p}^{2}} \epsilon^{i j k} E_{i}^{a} E_{j}^{b}\left(F_{a b k}+\frac{\ell_{p}^{2} V(\phi)}{3} \epsilon_{a b c} E_{k}^{c}\right)} .
$$

Combining (81), (91), and (12) one can show that the integrated Hamiltonian constraint is

$$
\mathcal{H}(\tilde{N})=\frac{1}{2 \ell_{p}^{2}} \int_{\mathcal{S}} \pi \mp \frac{1}{\sqrt{2} \ell_{p}^{2}} \int_{\mathcal{S}} \sqrt{-\frac{1}{\ell_{p}^{2}} \epsilon^{i j k} E_{i}^{a} E_{j}^{b}\left(F_{a b k}+\frac{\ell_{p}^{2} V(\phi)}{3} \epsilon_{a b c} E_{k}^{c}\right)} .
$$

We use $\phi$ to define a time coordinate ${ }^{2}$

$$
T:=\ell_{p}^{2} \phi
$$

with conjugate momentum

$$
\begin{gathered}
P:=\frac{1}{l_{p}^{2}} \int_{\mathcal{S}} \pi \\
\Rightarrow\{T, P\}=1
\end{gathered}
$$

so that setting the integrated constraint $\mathcal{H}(\tilde{N})=0$ in (13) implies

$$
H=P
$$

where we recognize

$$
H:= \pm \frac{\sqrt{2}}{\ell_{p}^{2}} \int_{\mathcal{S}} \sqrt{-\frac{1}{\ell_{p}^{2}} \epsilon^{i j k} E_{i}^{a} E_{j}^{b}\left(F_{a b k}+\frac{\ell_{p}^{2} V(T)}{3} \epsilon_{a b c} E_{k}^{c}\right)}
$$

\footnotetext{
${ }^{2}$ Note that slow-roll inflation [1] is achieved by a decreasing $\phi$ (i.e. $\phi$ is "rolling down the hill" of the potential). This means that the forward progression of time corresponds to a decreasing $T$.
} 
as the Hamiltonian that generates evolution in the parameter $T$. Herein we will use $T$ instead of $\phi$ and denote the scalar field potential as $V(T)$ to mean the original scalar field potential $V(\phi)$ evaluated at $\phi=T / \ell_{p}^{2}$.

To summarize, by choosing an appropriate coordinate gauge, we have been able transform the constrained Hamiltonian theory of general relativity into a theory whose dynamics are generated by a traditional Hamiltonian functional (18). This will be the starting point for reducing to homogeneous spacetimes and, eventually, quantizing to obtain a quantum theory for a homogeneous universe.

\section{Bianchi Class A Models}

We now proceed to specialize the Hamiltonian formalism of the previous section to consider only the so-called Bianchi class A models. The result will be a simpler theory that can immediately be quantized, providing a quantum framework to describe a homogeneous, inflationary universe.

A spatially homogeneous spacetime ${ }^{3}$ is characterized by a 3-dimensional symmetry group acting transitively on the spatial manifold $\mathcal{S}$. Such a group can be described by its algebra with structure constants $c_{J K}^{I}$ which can, in general, be written as

$$
c^{K}{ }_{I J}=M^{K L} \epsilon_{L I J}+\delta_{[I}^{K} A_{J]}
$$

where $M^{K L}$ and $A_{J}$ are any tensors satisfying

$$
M^{I J} A_{J}=0 .
$$

The Bianchi class A models are those in which $A_{J}=0$. This class consists of 6 models (up to isomorphism) and we further restrict our consideration to those 5 whose structure constants can be written in the form

$$
c_{J K}^{I}=n^{(I)} \epsilon_{J K}^{I}
$$

where the brackets around an index indicate that it is not to be summed over. Herein, we will use the term Bianchi class A to mean this subset of 5 models.

\footnotetext{
${ }^{3} \mathrm{~A}$ complete description of homogeneous spacetimes is given in [13] where the reader is referred to for a more detail derivation of the facts stated here.
} 
It can be shown 13] that one can always construct a basis of 1-form fields $\left\{\omega^{I}\right\}$ with dual vector fields $\left\{X_{I}\right\}$ which are left-invariant under the action of the symmetry group (i.e. a basis that does not change when acted upon by a group element from the left). Such a basis is uniquely determined by demanding that it satisfy the "curl" relations

$$
d \omega^{I}=-\frac{1}{2} c^{I}{ }_{J K} \omega^{J} \wedge \omega^{K}
$$

and that its Lie derivative along timelike vector fields orthogonal to the spatial slices vanish. It will prove advantageous to utilize such an invariant basis in order to simplify the calculations.

Following the procedure described in [14, 15], we expand the $A$ and $E$ fields in terms of these left-invariant bases. The connection and momentum can then be written as

$$
A_{a}^{i}(x, T)=c_{(I)}(T) \Lambda_{I}^{i} \omega_{a}^{I}, \quad E_{i}^{a}(x, T)=p^{(I)}(T) \Lambda_{i}^{I} X_{I}^{a}
$$

where $\Lambda \in S O(3)$ and the functions $\left\{c_{I}\right\}$ and $\left\{p^{I}\right\}$ are constant on a given spatial slice and only depend on the time variable (14). Please note that (23) is not the most general form of the homogeneous variables but is, in fact, a diagonalized form that is well suited to the study of Bianchi class A models. The $S O(3)$ matrices are, essentially, a rotation of the invariant basis introduced in order to obtain this diagonal form of the connection and momentum. These matrices are a statement of the gauge degrees of freedom in the $A$ and $E$ variables. For the computations that are to follow, we will often use the following properties of $S O(3)$ matrices:

$$
\Lambda_{I}^{i} \Lambda_{i}^{J}=\delta_{I}^{J}, \quad \epsilon_{i j k} \Lambda_{I}^{i} \Lambda_{J}^{j} \Lambda_{K}^{k}=\epsilon_{I J K} .
$$

In order to avoid infinite quantities arising from the (possible) infinitude of space, we consider a compact submanifold $\Sigma \subset \mathcal{S}$ of the full spatial manifold $\mathcal{S}$. Since the spatial manifold is, by definition, homogeneous, no generality is lost by this reduction. We parametrize this submanifold choice by defining a dimensionless parameter, $R$, such that

$$
R^{3}:=\frac{1}{\ell_{p}^{3}} \int_{\Sigma}
$$

where the volume element on $\Sigma$ is understood. The $\ell_{p}^{-3}$ normalization is simply an arbitrary length scale, chosen to be the Planck length. $R$ can then 
be considered a parameter of the model that measures the size of our sample of space.

The reduction (23) of the full theory to Bianchi class A models reduces general relativity from a Hamiltonian theory with an infinite number of degrees of freedom to one with only three degrees of freedom, $c_{1}, c_{2}$, and $c_{3}$ and their conjugate momenta $p^{1}, p^{2}$, and $p^{3}$. To determine their canonical relationship, we must first define $c_{I}$ and $p^{I}$ in terms of the variables $A_{a}^{i}$ and $E_{i}^{a}$. A convenient definition is

$$
\begin{aligned}
c_{I} & =\frac{1}{\ell_{p}^{3} R^{3}} \int_{\Sigma} A_{a}^{i} \Lambda_{i}^{(I)} X_{I}^{a} \\
p^{I} & =\frac{1}{\ell_{p}^{3} R^{3}} \int_{\Sigma} E_{i}^{a} \Lambda_{(I)}^{i} \omega_{a}^{I} .
\end{aligned}
$$

With these definitions we can use the relation (3) to compute the Poisson bracket

$$
\left\{c_{I}(T), \frac{R^{3} \ell_{p}}{i} p^{J}(T)\right\}=\delta_{I}^{J} .
$$

Hence, we recognize $\left(R^{3} \ell_{p} / i\right) p^{I}(T)$ as being the true canonical momenta to the variables $c_{I}(T)$. Of course, the Poisson bracket between different $c_{I}$ variables and between different $p^{J}$ variables respectively vanishes.

In order to write the diffeomorphism and Hamiltonian constraints in terms of the new phase space variables $\left(c_{I}, p^{J}\right)$, we must first calculate the curvature of the homogeneous connection

$$
F=d A+\frac{1}{2}[A, A]
$$

By expanding $A=A^{i} \tau_{i}$ in terms of the $s u(2)$ basis $\tau_{i}:=-\frac{i}{2} \sigma_{i}$ (where $\sigma_{i}$ are the Pauli matrices) and using the identities (22) and (24) we can work out the curvature components in the left-invariant basis to be

$$
F_{J L}^{i}=-n^{(I)} c_{(I)} \epsilon_{J L}^{I} \Lambda_{I}^{i}+c_{(J)} c_{(L)} \epsilon_{j k}^{i} \Lambda_{J}^{j} \Lambda_{L}^{k} .
$$

Here, the constants $\left\{n^{I}\right\}_{I=1}^{3}$ are the structure constants of the symmetry group defined in (21).

We can now use these curvature components and the homogeneous momentum field (23) to calculate the diffeomorphism constraint (17) in the left invariant basis. The result is the expression

$$
H_{I}=p^{(J)} \Lambda_{i}^{J}\left(-n^{(I)} c_{(I)} \epsilon_{I J}^{L} \Lambda_{I}^{L}+c_{(I)} c_{(J)} \epsilon_{j k}^{i} \Lambda_{I}^{j} \Lambda_{J}^{k}\right)
$$


which, by applying the first relation in (24), can be shown to vanish identically for all Bianchi class A models. So, by reducing our field variables to an intrinsically homogeneous and diagonalized form, we have also, at the same time, automatically satisfied the diffeomorphism constraint. Note that this result is not necessarily true for more general forms of the homogeneous variables.

An expression for the Hamiltonian (18) in terms of the new phase space variables can be obtained in a similar fashion. That is, by substituting the curvature (30) and the components of the homogeneous variables (23) into the Hamiltonian and applying the $S O(3)$ relations (24) one obtains the Hamiltonian

$$
H\left(c_{I}, p^{J} ; T\right)= \pm 2 R^{3}\left[p^{1} p^{2} c_{1} c_{2}\left(n^{3} \frac{c_{3}}{c_{1} c_{2}}-1-\frac{\ell_{p}^{2} V(T)}{3} \frac{p^{3}}{c_{1} c_{2}}\right)+\text { cyc. }\right]^{\frac{1}{2}} .
$$

Recall that $V(T)$ is the potential energy function for the scalar field $\phi=\ell_{p}^{-2} T$, kept as an arbitrary function throughout the paper.

In order to determine the reality condition (4) for the new phase space variables, one must, first, expand the three dimensional spin connection in terms of the $(S O(3)$ rotated) left invariant basis

$$
\Gamma_{a}^{i}=\Gamma_{(I)} \Lambda_{I}^{i} \omega_{a}^{I}
$$

where $\Gamma_{I}$ can be computed [16] to be

$$
\Gamma_{I}=\frac{1}{2}\left(n^{J} \frac{p^{K}}{p^{J}}+n^{K} \frac{p^{J}}{p^{K}}-n^{I} \frac{p^{J} p^{K}}{\left(p^{I}\right)^{2}}\right) \quad \text { (no summation on indices) }
$$

where $(I, J, K)$ are even permutations of $(1,2,3)$. The resulting reality conditions are then

$$
\begin{aligned}
c_{I}+\overline{c_{I}} & =2 \Gamma_{I} \\
p_{I}-\overline{p_{I}} & =0 .
\end{aligned}
$$

Due to the presence of the $n^{I}$ parameters in (34), we see that the reality conditions are dependent on which Bianchi model is chosen.

Our classical Hamiltonian theory for a homogeneous universe with a scalar field is now completely defined. We have phase space variables $c_{I}$ and $p^{I}$ with the symplectic structure (28), the reality conditions (35) and (36) and a time 
dependent Hamiltonian (32) that governs the dynamics. The definitions (23) then relate the phase space variables back to the Ashtekar-Sen variables from which we can then infer all of the information concerning the spacetime geometry. Since there are only a finite number of degrees of freedom and no constraints needing to be satisfied, one can, in principle, quantize this model in a straightforward manner following the traditional algorithm of quantizing classical Hamiltonian systems [17].

\subsection{Zero-Energy Classical Solution}

Before looking at the quantum theory, it would be very desirable to obtain a general solution to this classical Hamiltonian theory. As was seen in the calculation of the quantum solution to the flat, isotropic inflationary model in 10], such a classical solution can be crucial in finding a solution to the corresponding quantum theory. This will again be the case in the following section.

A first attempt at a general, classical solution may be made via HamiltonJacobi theory [18] in which we seek a Hamilton-Jacobi function $S\left(c_{1}, c_{2}, c_{3} ; T\right)$ such that

$$
p^{I}=\frac{i}{R^{3} \ell_{p}} \frac{\partial S}{\partial c_{I}}, \quad P=\frac{\partial S}{\partial T}
$$

where the constant coefficient in the first relation comes from the nonstandard Poisson bracket (28). Such a function is determined by equation (17) which now reads as a partial differential equation

$$
\begin{aligned}
\frac{\partial S}{\partial T} & =H\left(c_{I}, \frac{i}{R^{3} \ell_{p}} \frac{\partial S}{\partial c_{I}} ; T\right) \\
\Rightarrow \frac{\partial S}{\partial T} & =\frac{2 i}{\ell_{p}}\left[\frac{\partial S}{\partial c_{1}} \frac{\partial S}{\partial c_{2}} c_{1} c_{2}\left(n^{3} \frac{c_{3}}{c_{1} c_{2}}-1-\frac{i \ell_{p} V(T)}{3 R^{3} c_{1} c_{2}} \frac{\partial S}{\partial c_{3}}\right)+\begin{array}{c}
\text { cyc. } \\
\text { perm. }
\end{array}\right]^{\frac{1}{2}}(38)
\end{aligned}
$$

where we have chosen the positive root of the Hamiltonian. The determination of such a Hamilton-Jacobi function is equivalent to solving the equations of motion.

While we are not able to find a completely general solution to (38) for arbitrary values of $n^{I}$, we are able to find a "zero-energy" function $S_{0}\left(c_{1}, c_{2}, c_{3} ; T\right)$, related to the phase space variables as in (37), such that $H=0$. The determining differential equation is the vanishing of the right hand side of (38) 
which does indeed vanish if

$$
\frac{\partial S_{0}}{\partial c_{K}}=\frac{3 i R^{3}}{\ell_{p} V}\left(c_{I} c_{J}-n^{(K)} c_{K}\right)
$$

where $(I, J, K)$ are cyclic permutations of $(1,2,3)$. Such a zero-energy function is given as

$$
S_{0}\left(c_{1}, c_{2}, c_{3} ; T\right)=\frac{3 i R^{3}}{\ell_{p} V}\left(c_{1} c_{2} c_{3}-\frac{1}{2} n^{I} c_{I}^{2}\right)
$$

plus an irrelevant constant. It is clear that this function does not solve the full Hamilton-Jacobi equation (38) since the time derivative of $S_{0}$ does not vanish for arbitrary potentials $V$. Of course this would be the general solution for the case where $V$ is just the cosmological constant with no time dependence.

As we will see in the next section (and as was seen in [10]), such zeroenergy functions are often a useful basis for an ansatz that can be used to find a general solution to the Hamilton-Jacobi equation. While such a strategy proved useful for the Bianch type I model considered in the next section, it is not clear how to successfully proceed with such a strategy for general Bianchi class A models.

\section{Bianchi Type I Classical and Quantum So- lutions}

We now turn our attention to the simplest, specific class A model: Bianchi I. The symmetry group of Bianchi $\mathrm{I}$ is the three dimensional translation group which is Abelian so the structure constants vanish and we have $n^{I} \equiv 0$. Furthermore, because of this commutativity, we may use the coordinate 1forms $d x^{I}$ as our left-invariant 1-forms,

$$
\omega^{I}=d x^{I}
$$

which clearly satisfy the curl relations (22).

Since the structure constants vanish for the model under consideration, it is clear that the 3 dimensional spin connection (34) also vanishes and so the reality conditions on the configuration space variables read

$$
c_{I}=-\overline{c_{I}}
$$


which can trivially be satisfied by defining new variables

$$
c_{I}^{\text {new }}:=-i c_{I}^{\text {old }}
$$

where $c_{I}^{\text {new }}$ can now be taken to be purely real (i.e. $c_{I}^{\text {old }}$ are purely imaginary in Bianchi I). Herein, we will use, simply, $c_{I}$ to denote the new, purely real variables. Of course, given the trivial reality conditions (36) on $p^{I}$, the conjugate momenta can be taken to be purely real. With these new $c_{I}$ variables, the Poisson bracket relation (28) between the phase space variables now reads

$$
\left\{c_{I}, p^{J}\right\}=\frac{1}{R^{3} \ell_{p}} \delta_{I}^{J} .
$$

We can now adapt the derivation of the previous section to this specific model. Using the real variables and $n^{I} \equiv 0$ in the Hamiltonian (32) we get the Bianchi I Hamiltonian

$$
H\left(c_{I}, p_{J} ; T\right)=2 R^{3}\left[p^{1} p^{2} c_{1} c_{2}\left(1-\frac{\ell_{p}^{2} V(T)}{3} \frac{p^{3}}{c_{1} c_{2}}\right)+\begin{array}{c}
\text { cyc. } \\
\text { perm. }
\end{array}\right]^{\frac{1}{2}}
$$

where, again, we have chosen the positive root. Given the slightly different Poisson bracket (44) for the real variables, a Hamilton-Jacobi function $S$ is now defined as

$$
p^{I}=\frac{1}{R^{3} \ell_{p}} \frac{\partial S}{\partial c_{I}}, \quad P=\frac{\partial S}{\partial T}
$$

and the corresponding Hamilton-Jacobi equation $P=H$ reads

$$
\frac{\partial S}{\partial T}=\frac{2}{\ell_{p}}\left[\frac{\partial S}{\partial c_{1}} \frac{\partial S}{\partial c_{2}} c_{1} c_{2}\left(1-\frac{\ell_{p} V(T)}{3 R^{3} c_{1} c_{2}} \frac{\partial S}{\partial c_{3}}\right)+\begin{array}{c}
\text { cyc. } \\
\text { perm. }
\end{array}\right]^{\frac{1}{2}} .
$$

To obtain a classical solution for this model, we begin with the zero-energy solution (40)

$$
S_{0}=\frac{3 R^{3}}{\ell_{p} V} c_{1} c_{2} c_{3}
$$

and take the ansatz

$$
S_{u}:=\frac{3 R^{3}}{\ell_{p} V} c_{1} c_{2} c_{3}(1+u(T))
$$


where $u(T)$ is a complex function of $\mathrm{T}$ alone. Upon substituting this ansatz into the Hamilton-Jacobi equation (47) we find that the $c_{I}$ variables cancel out on both sides and we are left with an ordinary differential equation in $u$

$$
\dot{u}=\frac{\dot{V}}{V}(1+u)+\frac{2 i}{\ell_{p}}(1+u) \sqrt{3 u}
$$

where the dot denotes differentiation with respect to $T$. Since this is an ordinary, differential equation, there exists a one (complex) dimensional solution space and, therefore, we have obtained a one-parameter family of solutions to the classical Bianchi I model via the Hamilton-Jacobi function (49).

Armed with a solution to the classical model we can now proceed to build a quantum Bianchi I framework following the usual quantization procedure. Since $c_{I}$ and $p^{I}$ are real valued, we take the corresponding operators $\hat{c}_{I}$ and $\hat{p}^{J}$ to be Hermitian

$$
\left(\hat{c}_{I}\right)^{\dagger}=\hat{c}_{I}, \quad\left(\hat{p}^{I}\right)^{\dagger}=\hat{p}^{I}
$$

The canonical commutation relations between these operators follow from the classical Poisson brackets and are given as

$$
\begin{aligned}
{\left[\hat{c}_{I}, \hat{p}^{J}\right] } & =i\left\{c_{I}, p^{J}\right\}=\frac{i}{R^{3} \ell_{p}} \delta_{I}^{J}, \\
{\left[\hat{c}_{I}, \hat{c}_{J}\right] } & =i\left\{c_{I}, c^{J}\right\}=0 \\
{\left[\hat{p}^{I}, \hat{p}^{J}\right] } & =i\left\{p_{I}, p^{J}\right\}=0 .
\end{aligned}
$$

We can represent the vectors in the Hilbert space as functions $\Psi: \mathbb{R}^{3} \rightarrow \mathbb{C}$ of the three configuration space variables $c_{1}, c_{2}$, and $c_{3}$. In this representation, the phase space operators act as

$$
\hat{c}_{I} \Psi=c_{I} \Psi, \quad \hat{p}^{I} \Psi=-\frac{i}{R^{3} \ell_{p}} \frac{\partial}{\partial c_{I}} \Psi .
$$

It is easy to check that these satisfy the above commutation relations.

The next step in this procedure is to construct a Hamiltonian operator corresponding to the classical expression (45). In terms of the classical variables, we may order the terms of the Hamiltonian (45) as

$$
H=2 R^{3} c_{1} c_{2} c_{3}\left[\frac{1}{c_{2}\left(c_{3}\right)^{2}} p^{1} \frac{1}{c_{1}} p^{2}\left(1-\frac{\ell_{p}^{2} V}{3 c_{1} c_{2}} p^{3}\right)+\begin{array}{c}
\text { cyc. } \\
\text { perm. }
\end{array}\right]^{\frac{1}{2}} .
$$


Using the operator representation (55) we can construct the corresponding operator as

$$
\hat{H}=-\frac{2 i}{\ell_{p}} c_{1} c_{2} c_{3}\left[\frac{1}{c_{2}\left(c_{3}\right)^{2}} \frac{\partial}{\partial c_{1}} \frac{1}{c_{1}} \frac{\partial}{\partial c_{2}}\left(1+\frac{i \ell_{p} V}{3 R^{3} c_{1} c_{2}} \frac{\partial}{\partial c_{3}}\right)+\begin{array}{c}
\text { cyc. } \\
\text { perm. }
\end{array}\right]^{\frac{1}{2}} .
$$

Our goal now is to find a solution $\Psi\left(c_{1}, c_{2}, c_{3} ; T\right)$ to the resulting Schrödinger equation

$$
i \frac{\partial \Psi}{\partial T}=\hat{H} \Psi
$$

We introduce the semi-classical state

$$
\Psi_{u}:=e^{i S_{u}}=e^{\frac{3 i R^{3}}{\ell_{p} V} c_{1} c_{2} c_{3}(1+u)}
$$

where $S_{u}$ is the classical Hamilton-Jacobi solution (49). If we denote the operator under the square root of (157) as $\hat{\Omega}$ so that

$$
\hat{H}=-\frac{2 i}{\ell_{p}} c_{1} c_{2} c_{3} \sqrt{\hat{\Omega}}
$$

then it can be shown by direct computation that $\Psi_{u}$ is an eigenvector of $\hat{\Omega}$ with time dependent eigenvalue:

$$
\begin{aligned}
\hat{\Omega} \Psi_{u} & =3 u\left(\frac{3 R^{3}}{\ell_{p} V}(1+u)\right)^{2} \Psi_{u} \\
\Rightarrow \sqrt{\hat{\Omega}} \Psi_{u} & =\sqrt{3 u\left(\frac{3 R^{3}}{\ell_{p} V}(1+u)\right)^{2}} \Psi_{u} .
\end{aligned}
$$

Hence, the corresponding action of the Hamiltonian operator (60) can immediately be determined as

$$
\hat{H} \Psi_{u}=-\frac{6 R^{3} i}{\ell_{p}^{2} V} c_{1} c_{2} c_{3}(1+u) \sqrt{3 u} \Psi_{u} .
$$

The surprising fact is that the semi-classical state (59) is, in fact, a full solution to the Schrödinger equation (58). To see this, we compute the time derivative of $\Psi_{u}$ to be

$$
\frac{\partial \Psi_{u}}{\partial T}=i \frac{3 R^{3}}{\ell_{p} V} c_{1} c_{2} c_{3}\left(-\frac{\dot{V}}{V}(1+u)+\dot{u}\right) \Psi_{u}
$$


and, upon substitution of the differential equation (50), this becomes

$$
\frac{\partial \Psi_{u}}{\partial T}=-\frac{6 R^{3}}{\ell_{p}^{2} V} c_{1} c_{2} c_{3}(1+u) \sqrt{3 u} \Psi_{u} .
$$

Comparison of this with the action of the Hamiltonian operator (63) clearly shows that $\Psi_{u}$ solves Schrödinger's equation (58). Hence, equation (59) gives a one parameter family of quantum states for an inflationary universe.

\subsection{Normalizability and the Relationship with the Ko- dama State}

A famous solution to the quantum constraint equations of quantum gravity with a cosmological constant is the so-called Kodama state [11. For a general (i.e. non-homogeneous) connection, it can be written as

$$
\Psi_{K}[A]:=\mathcal{N} e^{-i S_{C S}[A]}=\mathcal{N} e^{\frac{3}{2 \Lambda \ell_{p}^{2}} \int_{\mathcal{S}} Y_{C S}[A]}
$$

where $Y_{C S}[A]$ is the Chern-Simons invariant

$$
Y_{C S}[A]:=\frac{1}{2} \operatorname{Tr}\left(A \wedge d A+\frac{2}{3} A \wedge A \wedge A\right)
$$

and $\mathcal{N}$ is a normalization factor depending only on the topology of the manifold [19]. Similar to the homogeneous, inflationary solution found above, the Kodama state is also a semi-classical state corresponding to the HamiltonJacobi functional

$$
S_{C S}[A]=\frac{3 i}{2 \Lambda G} \int_{\mathcal{S}} Y_{C S}[A]
$$

which solves the classical constraint equations of gravity.

One of the motivations for studying our inflationary model was to try to find states similar to the Kodama state (66) in a framework in which the state's properties could more easily be calculated. In this section, we investigate the similarities between the Kodama state (66) and our inflationary state (59) and discuss the relevant properties of the latter.

In order to see what the Kodama state looks like in a Bianchi spacetime, we need to compute the Chern-Simons invariant (67) using the homogeneous 
connection (23) . We do this by explicitly expanding the connection $A$ as $A^{i} \tau_{i}$ (recall that $\tau_{i}=-\frac{i}{2} \sigma_{i}$ ) to obtain

$$
Y_{C S}=\frac{1}{4}\left(n^{i} c_{i}^{2}-2 c_{1} c_{2} c_{3}\right) \omega^{1} \wedge \omega^{2} \wedge \omega^{3}
$$

where $\omega^{I}$ are the left-invariant basis 1-forms. Substituting this into the Hamilton-Jacobi functional (68) we get

$$
S_{C S}=\frac{3 i R^{3} \ell_{p}}{4 \Lambda}\left(\frac{1}{2} n^{i} c_{i}^{2}-c_{1} c_{2} c_{3}\right)
$$

which is the same as the zero-energy Hamilton-Jacobi function $S_{0}$ (40) with the scalar potential $V(T)$ chosen to be constant. Of course, this similarity is not so surprising. The $S_{C S}$ function was chosen so that the Hamiltonian constraint vanished while $S_{0}$ is defined as that function which causes the Hamiltonian (32), which is the integral of the square root of the Hamiltonian constraint, to vanish.

Using this result we can specialize the Kodama state to Bianchi I to obtain

$$
\Psi_{K}=\mathcal{N} e^{-i S_{C S}}=\mathcal{N} e^{i \frac{3 R^{3} \ell_{p}}{4 \Lambda} c_{1} c_{2} c_{3}} .
$$

Comparing this with the inflationary state $\Psi_{u}$ from equation (59) we see they are the same state up to the time varying function $u(T)$ (with the appropriate choice of constant potential $V(T)$ ). We also emphasize, once again, that both the Kodama state $\Psi_{K}$ and our new state $\Psi_{u}$ are semi-classical states as well as full solutions to their respective theories (the former solves the constraint equations while the latter solves Schrödinger's equation). These similarities suggest that $\Psi_{u}$ is a modified Kodama state; it is the analogue of the Kodama state when one couples a scalar field to the quantum gravitational field.

Due to the fact that the configuration space variables $c_{I}$ are real, we may take the inner product between two states $|\Psi\rangle$ and $|\Phi\rangle$ to be

$$
\langle\Psi \mid \Phi\rangle=\int_{\mathbb{R}^{3}} d c_{1} d c_{2} d c_{3} \bar{\Psi}\left(c_{1}, c_{2}, c_{3}\right) \Phi\left(c_{1}, c_{2}, c_{3}\right)
$$

where, as usual, $\Psi\left(c_{1}, c_{2}, c_{3}\right):=\left\langle c_{1}, c_{2}, c_{3} \mid \Psi\right\rangle$ and $\left|c_{1}, c_{2}, c_{3}\right\rangle$ are the simultaneous eigenvectors of the $\hat{c}_{I}$ operators. Using this inner product, it is clear that both the Kodama state (71) and the modified Kodama state (59) are both continuously normalizable (also called delta function normalizable) because they are simply a phase. 
We now recall the fact there there is a one parameter family of solutions $u(T)$ to the differential equation (50), each one of which corresponding to a different state $\Psi_{u}$. If we label each solution $u_{w}(T)$ by its value $w:=u\left(T_{0}\right)$ at some initial time $T=T_{0}$ then we may construct (truly) normalizable wavefunctions by making "wave packets" of these solutions

$$
\Psi_{f}\left(c_{1}, c_{2}, c_{3} ; T\right):=\int d w f(w) \Psi_{u_{w}}=\int d w f(w) e^{i \frac{R^{3}}{\ell_{p} V} c_{1} c_{2} c_{3}\left(1+u_{w}\right)}
$$

where $f(w)$ is a normalizable function with support on a suitable interval similar to that discussed in $[10]^{4}$. By normalizable, we mean that

$$
\int d w|f(w)|^{2}<\infty
$$

This type of normalization is not possible in the Kodama state (71) as there is no such one-parameter family of solutions to take wave packets of.

Here we see that, by introducing a scalar field into our model, we are able to describe the gravitational field (encoded by the configuration space variables $c_{I}$ ) with respect to the scalar field $T \approx \phi$. In this framework, the modified Kodama state (59), which is only continuously normalizable in the standard inner product, is able to form a normalizable state by making wave packets.

\section{The Spatially Flat, Isotropic Model: A Spe- cial Case of Bianchi I}

Before discussing the implications of the above results, we wish to write the solution of the previous section specifically for a spatially flat and isotropic spacetime. In this case, the real, homogeneous configuration space variables are all equal

$$
c_{1}=c_{2}=c_{3}=: A
$$

as are their conjugate momenta

$$
p^{1}=p^{2}=p^{3}=: E .
$$

\footnotetext{
${ }^{4}$ In that paper [10], it was shown that that the value of the function $u(T)$ is constrained such that the metric remain real and maintain a Lorentzian signature. Hence, $w$ is similarly constrained to such values.
} 
The Hamiltonian (45) then reads

$$
H=2 R^{3} A^{3}\left[3 \frac{1}{A^{2}} E \frac{1}{A^{2}} E\left(1-\frac{\ell_{p}^{2} V}{3 A^{2}} E\right)\right]^{\frac{1}{2}}
$$

where we have written the terms in the order in which they will be written in the corresponding Hamiltonian operator.

We will now write the classical and quantum solutions without much commentary as the derivation is completely analogous to that of the previous section. The classical Hamilton-Jacobi function is

$$
S_{v}(A, T)=\frac{R^{3}}{\ell_{p} V(T)} A^{3}(1+v(T))
$$

where $v$ is a function of $T$ alone and satisfies the ordinary differential equation

$$
\dot{v}=\frac{\dot{V}}{V}(1+v)+\frac{6 i}{\ell_{p}}(1+v) \sqrt{3 v} .
$$

The representation of the $\hat{A}$ and $\hat{E}$ operators can be taken as

$$
\hat{A} \Psi=A \Psi, \quad \hat{E} \Psi=-\frac{i}{R^{3} \ell_{p}} \frac{\partial}{\partial A} \Psi
$$

and the corresponding solution to Schrödinger's equation (158) is

$$
\Psi_{v}:=e^{i S_{v}}=e^{\frac{i R^{3}}{\ell_{p} V} A^{3}(1+v)}
$$

which, as in (59), is also the semi-classical state.

This wavefunction $\Psi_{v}$ is different from the wavefunction derived in [10] using the same framework. The difference is due to the use of different operator orderings in the Hamiltonian. The benefit of the solution in this paper is that it stems from the more general Bianchi I solution (59) whereas the solution described in [10] cannot be generalized. Furthermore, $\Psi_{v}$ is a semi-classical state that is also a full solution to Schrödinger's equation and, in this respect, is more closely related to the Kodama state, as was discussed in the previous section. The wavefunction of [10] does not share this property but is in fact written as a modified semi-classical state based on the classical solution (78). 


\subsection{The Spectrum of the Scale Factor}

As a simple example of how one may go about gaining information from such a quantum cosmological framework, we can ask what the spectrum of eigenvalues is for the operator corresponding to the classical scale factor in a flat, isotropic universe. Recalling that $E_{i}$ is just the densitized triad of the spatial manifold, one can easily show that the metric takes the form

$$
d s^{2}=-N^{2} d T^{2}+E(T)\left(\left(d x^{1}\right)^{2}+\left(d x^{2}\right)^{2}+\left(d x^{3}\right)^{2}\right)
$$

where $N$ is the undensitized lapse function described in detail in [10]. Hence, $E(T)$ is the square root of the scale factor.

Since the operator $\hat{E}$ is simply a (scaled) derivative operator (80), its eigenvalues are analogous to the familiar momentum eigenvalues from ordinary quantum mechanics. The eigenvectors of $\hat{E}$ are given by

$$
\psi_{E}(A)=e^{i E R^{3} \ell_{p} A}
$$

and are labelled by the real eigenvalues $E$. As in ordinary quantum mechanics, if the domain of $A$ is $\mathbb{R}$ (as was assumed in [10] and in this paper), then the spectrum of $\hat{E}$ is continuous. This runs contrary to the prediction of loop quantum gravity [2] and, more specifically, to the cosmological predictions of Martin Bojowald [20], that the spectrum of volume eigenvalues be quantized. If the scale factor had discrete eigenvalues, it would suggest that the universe cannot evolve continuously in a classical manner but must proceed by some quantum evolution. The fact that we do not find this in our model may be a hint that our approach to quantum cosmology is too naive.

Another possibility that this result may suggest is that the domain of $A$ should not be taken as $\mathbb{R}$ but as a finite interval. If quantum states were required to have periodic boundary conditions on such a finite interval then the spectrum would be quantized just as angular momentum is quantized in ordinary quantum mechanics. Further study is required in order to resolve this issue.

\section{Discussion and Future Projects}

We have shown that the incorporation of a scalar field into the AshtekarSen formalism of general relativity allows for a choice of time gauge which leads to a standard Hamiltonian theory. By restricting this formalism to a 
class of homogeneous spacetimes (a subclass of Bianchi class A), we obtain a Hamiltonian theory with a finite number of degrees of freedom that describes a homogeneous universe filled with a scalar field. The finite number of degrees of freedom allows us to straightforwardly quantize to obtain a corresponding quantum framework to describe inflationary cosmology.

By looking strictly at the Bianchi I model we are able to find a oneparameter family of classical solutions. That is, for every solution $u(T)$ of the differential equation (50) we obtain a homogeneous spacetime solution given by the Hamilton-Jacobi function $S_{u}$ (49). The solutions $u(T)$ also label a class of quantum states (59) that are full solutions to the corresponding quantum theory. These quantum states are the semi-classical states $e^{i S_{u}}$ corresponding to the classical solutions given by $S_{u}$. We considered flat and isotropic spacetimes as a special case of Bianchi I in order to provide an alternative solution to that give in 10.

We close our discussion with a brief review of what is left to be done and some possible directions this work may go in the future.

Reality conditions and a physical inner product. In the Bianchi class A framework of section 3, the classical configuration space variables $c_{I}$ were, in general, complex numbers. A quantization of such a complex Hamiltonian theory requires a specially constructed inner product so that expectation values of real quantities turn out to be real. This problem was avoided in the Bianchi I case because the 3-dimensional spin connection vanished and we were able to redefine the theory in terms of purely real variables. In such a framework, the standard inner product suffices. For other Bianchi models, the spin connection does not vanish identically, the $c_{I}$ cannot be taken to be real, and a new inner product will need to be defined. In order to have a well-defined quantum framework for the class A models, such an inner product will need to be found that is applicable to all models.

Hermitian ordering of the Hamiltonian. Throughout this paper, and in [10], we have used an operator ordering that leads to a non-Hermitian Hamiltonian operator. As is well known, a Hamiltonian must be Hermitian in order for probability to be conserved. Hence, it may be required to solve the quantum theory using a Hermitian ordering of the Hamiltonian in order to obtain a probabilistic interpretation for the wavefunction solution. If this is, indeed, required, it is hoped that the techniques used in this paper will lead to a similar solution to the Schrödinger equation for the new Hamiltonian. 
Such a solution has been searched for without any success to date.

However, given the difficulty in interpreting any wavefunction of the universe it is not clear that the ordinary interpretation of quantum mechanics can be applied to a theory of quantum cosmology. For this reason, we have gone ahead with the non-Hermitian Hamiltonian operator as it leads to an exact solution analogous to the Kodama state. Of course, such a solution will also be a solution to the Schrödinger equation using a Hermitian Hamiltonian at the semi-classical level.

Numerical analysis. All of the cosmological solutions based on the framework of this paper have depended on the solution to an ordinary differential equation (e.g. equation (50) ) which cannot be written in closed form. Some initial numerical analysis has been done on such equations [10] but, to fully understand the dynamics of the corresponding quantum solutions $\Psi_{u}(\mathbf{5 9})$, it will be necessary to better understand the dynamics of $u(T)$. It seems that the best way to do this is through computational integration of the differential equation.

Another motivation for the research discussed in this paper was to examine the dynamics of a quantum Bianchi IX universe in which the symmetry group of space is $S U(2)$. The study of classical spacetimes suggests that the dynamics of the spatial geometry of a general manifold close to a singularity (such as the big bang singularity) can accurately be modelled by a Bianchi IX spacetime [21 and that the evolution of such a Bianchi IX model is chaotic [13, 22. A recent result of the loop quantum cosmology program [8] is that loop quantum gravity effects become important close to such classical singularities and that such effects curb the chaotic evolution of the quantum Bianchi IX model which lead to a more regular evolution of the spatial geometry in such regimes [16, 23].

It will be interesting to study the Bianchi IX model in our framework to see whether or not the evolution is chaotic close to classical singularities. Since an exact wavefunction solution was not found for the Bianchi IX model, it may be necessary to evolve an initial state numerically using the Bianchi IX Hamiltonian operator (the operator version of equation (32) with $n^{I} \equiv 1$ for all $I$ ) in order to proceed with this analysis.

Spatial perturbations and the cosmic microwave background. The greatest success of standard, inflationary cosmology is the accurate prediction of the spectrum of perturbations observed in the cosmic microwave 
background (CMB) 1]. Due to the exponential expansion of space during the inflationary epoch and the dynamics of quantum scalar fields in such spacetimes, scales well below the Planck length are expanded to macroscopic scales in most inflationary models 24]. Since many believe that low energy physics no longer applies at such small scales, the precise measurement of the CMB may provide the first observational evidence for quantum gravity. Indeed, a recent semi-classical analysis [25] suggests loop quantum gravitational effects may leave an observational signature in the CMB.

The general framework described in this paper may prove to be an advantageous starting point for a more accurate calculation of the CMB spectrum that incorporates the quantum nature of the gravitational field. While very little work has been done in this regard, one possible direction is to follow an analogous linearization procedure to that described in 26] about one of our Bianchi models. In that paper, the authours are able to define an inner product which is necessary for any computation. While they find that the linearized Kodama state is non-normalizable (in the Lorentzian theory) it is hoped that the modifications introduced by the scalar field and the choice of time gauge described in this paper will lead to a normalizable state. This was indeed the case for the purely homogeneous states, as described in section 4.1. The successful calculation of the CMB spectrum from a theory of quantum gravity would then give us an observable prediction which could either support or falsify our approach to quantum cosmology.

\section{Acknowledgements}

The authour would like to thank Lee Smolin and Stephon Alexander for their continued collaboration in this research program and for useful discussions during the course of this work.

\section{References}

[1] A.R. Liddle and D.H. Lyth Cosmological Inflation and Large-Scale Structure Cambridge University Press: Cambridge (2000).

[2] C. Rovelli Quantum Gravity To be published by Cambridge University Press in October, 2004. Available online at http://www.cpt.univ-mrs.fr/〜 rovelli/book.pdf. 
[3] T. Thiemann Introduction to Modern Canonical Quantum General Relativity arXiv:gr-qc/0110034.

[4] C. Rovelli "Loop Quantum Gravity", Living Rev. Relativity 1, 1 (1998). [Online article]: cited on November 14, 2018, http://www.livingreviews.org/lrr-1998-1.

[5] L. Smolin Quantum Gravity with a Positive Cosmological Constant arXiv:hep-th/0209079.

[6] A. Ashtekar New Variables for Classical and Quantum Gravity Phys. Rev. Lett. 572244 (1986); New Hamiltonian Formulation of General Relativity Phys. Rev. D36 1587 (1987).

[7] A. Ashtekar Lectures on Non-Perturbative Canonical Gravity World Scientific: Singapore (1991).

[8] M. Bojowald and H.A. Morales-Técotl Cosmological Applications of Loop Quantum Gravity Lect. Notes Phys. 646, 421 (2004) arXiv:gr-qc/0306008.

[9] M. Bojowald ABsence of a Singularity in Loop Quantum Cosmology Phys. Rev. Lett. 865227 (2001) arXiv:gr-qc/0102069.

[10] S. Alexander, J. Malecki and L. Smolin Loop Quantum Gravity and Inflation, Phys. Rev. D70 044025 (2004) arXiv:hep-th/0309045.

[11] H. Kodama, Prog. of Theo. Phys. 80, 1024 (1988); Phys. Rev. D D42 2548 (1990).

[12] P.A.M. Dirac Lectures on Quantum Mechanics Belfer Graduate School of Science Monographs 2, Yeshiva University Press: New York (1964).

[13] M.P. Ryan and L.C. Shepley Homogeneous Relativistic Cosmologies Princeton University Press: Princeton (1975).

[14] H. Kodama Specialization of Ashtekar's Formalism to Bianchi Cosmology, Progr. of Theo. Phys. 80, 6, 1024 (1988).

[15] M. Bojowald Homogeneous Loop Quantum Cosmology Class. Quant. Grav. 202595 (2003) arXiv:gr-qc/0303073. 
[16] M. Bojowald, G. Date and K. Vandersloot Homogeneous Loop Quantum Cosmology: The Role of the Spin Connection Class. Quant. Grav. 21 1253 (2004) arXiv:gr-qc/0311004.

[17] P.A.M. Dirac The Principles of Quantum Mechanics, 4th Ed. Oxford University Press: Oxford (1958).

[18] H. Goldstein, C. Poole and J. Safko Classical Mechanics Addison Wesley: San Fransisco (2002).

[19] C. Soo Wave Function of the Universe and Chern-Simons Perturbation Theory Class. Quant. Grav. 191051 (2002) arXiv:gr-qc/0109046|.

[20] M. Bojowald Loop Quantum Cosmology: II. Volume Operator Class. Quant. Grav. 171509 (2000) arXiv:gr-qc/9910104.

[21] V.A. Belinskii, I.M. Khalatnikov and E.M. Lifshitz A General Solution of the Einstein Equations with a Time Singularity Adv. in Phys. 31, 6, 639 (1982).

[22] T. Damour, H. Henneaux and H. Nicolai Cosmological Billiards, Class. Quant. Grav. 20, R145 (2003) arXiv:hep-th/0212256.

[23] M. Bojowald and G. Date A Non-Chaotic Quantum Bianchi IX Universe and the Quantum Structure of Classical Singularities Phys. Rev. Lett. 92071302 (2004) arXiv:gr-qc/0311003.

[24] J. Martin and R. Brandenberger Trans-Planckian Problem of Inflationary Cosmology Phys. Rev. D 63123501 (2001) arXiv:hep-th/0005432; Dependence of the Spectra of Fluctuations in Inflationary Cosmology on Trans-Planckian Physics Phys. Rev. D 68063513 (2003) arXiv:hep-th/0305161.

[25] S. Tsujikawa, P. Singh and R. Maartens Loop Quantum Gravity Effects on Inflation and the $C M B$ arXiv:astro-ph/0311015.

[26] L. Friedel and L. Smolin The Linearization of the Kodama State arXiv:hep-th/0310224. 